\title{
Criminologie
}

\section{Droits des détenus, normalisation et moindre éligibilité}

\section{Dan Kaminski}

Volume 43, numéro 1, printemps-été 2010

Les jeunes et la rue

URI : https://id.erudit.org/iderudit/044057ar

DOI : https://doi.org/10.7202/044057ar

Aller au sommaire du numéro

Éditeur(s)

Les Presses de l'Université de Montréal

ISSN

0316-0041 (imprimé)

1492-1367 (numérique)

Découvrir la revue

Citer cet article

Kaminski, D. (2010). Droits des détenus, normalisation et moindre éligibilité. Criminologie, 43(1), 199-226. https://doi.org/10.7202/044057ar

\section{Résumé de l'article}

À l'invitation de la revue Criminologie pour son quarantième anniversaire, cette conférence, donnée le 7 novembre 2008, interroge la promotion contemporaine des droits des détenus dans un contexte sociopolitique qui fait la part belle à la moindre éligibilité. Une effectivité réellement normalisatrice des droits des détenus demande la levée d'obstacles politiques et culturels qui en appauvrissent aujourd'hui la portée. d'utilisation que vous pouvez consulter en ligne.

https://apropos.erudit.org/fr/usagers/politique-dutilisation/ 


\section{Droits des détenus, normalisation et moindre éligibilité}

Dan Kaminski

Professeur

École de criminologie, Université catholique de Louvain

Centre de recherche interdisciplinaire sur la déviance et la pénalité (CRID\&P)

dan.kaminski@uclouvain.be

RÉSUMÉ - À l'invitation de la revue Criminologie pour son quarantième anniversaire, cette conférence, donnée le 7 novembre 2008, interroge la promotion contemporaine des droits des détenus dans un contexte sociopolitique qui fait la part belle à la moindre éligibilité. Une effectivité réellement normalisatrice des droits des détenus demande la levée d'obstacles politiques et culturels qui en appauvrissent aujourd'hui la portée.

MOTS-CLÉS - Droits des détenus, normalisation, moindre éligibilité.

«Non seulement mais aussi seulement» Georges Aperghis, Avis de tempête ${ }^{1}$

\section{Introduction}

J'aurais voulu traiter aujourd'hui des droits des détenus comme une conquête relative, de leur inscription dans le contexte social contemporain, du dilemme qu'impose la critique de l'institution totale quand on examine la totalisation de la «liberté», et des rapports complexes entre normalisation et moindre éligibilité. C'était trop pour une seule fête, et la réduction de mon propos au dernier thème cité me paraît encore bien mal achevée. Je voudrais commencer par remercier les animateurs de la

1. Georges Aperghis est un compositeur contemporain de théâtre musical, de musique de concert et d'opéra. Avis de tempête est un opéra pour soprano, deux barytons, danseuseactrice, électronique, vidéo guitare électrique et deux claviers, composé en 2004 sur un livret de Georges Aperghis et Peter Szendy. Voir www.aperghis.com. 
revue Criminologie qui, incontestablement, m'ont fait travailler ${ }^{2}$. J'ai bénéficié ainsi du double honneur de publier une petite contribution dans le numéro du $40^{\mathrm{e}}$ anniversaire et d'en présenter une autre, orale, aujourd'hui. Je suis donc ravi, confus, fourbu et angoissé. L'honneur exige en effet d'être à la hauteur et ne confère aucun droit. Cette dernière assertion, moraliste, me place déjà au cœur du sujet...

Mon propos tente de s'inscrire dans une proposition théorique stimulante de Gilles Chantraine (2004). Contre le carcéralo-centrisme [...], l'analyse de la privation de liberté doit être articulée à « une histoire de la "liberté" elle-même», ou, plus précisément, des assujettissements «qui se forment en son nom ${ }^{3} »$. Chantraine (2004) continue:

Foucault a décrit une prison correctionnaliste, à une époque où la liberté des Lumières allait de pair avec la normalité, d'où l'essor et le développement des grandes disciplines sociales qui ont fait l'objet de la critique du philosophe. Aujourd'hui, après une période durant laquelle la liberté a été fortement connotée aux conceptions de la solidarité sociale, elle apparaît davantage articulée aux injonctions à l'individuation, à l'autonomie, à la réalisation de soi dans le travail, à l'introspection psychologique et au bonheur consumériste. Un jeu permanent entre l'ordinaire (formes partagées intra et extra muros des techniques de gouvernement) et l'extraordinaire doit permettre de renouveler et de complexifier la critique.

Les trois notions réunies dans mon titre, dont je vais rapidement préciser ici la signification, n'ont pas la même valeur ni la même origine, ni encore le même âge. Elles sont associées pour permettre une réouverture de l'espace, un renouvellement de la critique. La reconnaissance de droits aux détenus permet de transformer la vie pénitentiaire (la situation d'enfermement et les rapports sociaux qui s'y déploient) en fournissant aux détenus des ressources juridiques opposables à l'administration. La normalisation (des conditions de la vie carcérale) est le concept qui rend compte de l'objectif politique auquel s'indexent notamment les droits des détenus: il s'agit de tendre vers une prison dans laquelle les conditions de vie ressemblent le plus possible aux conditions de la vie libre. La moindre éligibilité est en quelque sorte le

2. Je voudrais aussi remercier Françoise Tulkens (juge belge à la Cour européenne des droits de l'homme) et Sonja Snacken (professeure de criminologie à la Vrije Universiteit Brussel) pour leur lecture attentive de la version écrite de cette conférence et les commentaires qu'elles ont bien voulu y apporter. Aucune d'elles n'assumera la moindre responsabilité pour l'oreille que j'ai prêtée à ces derniers.

3. Dans le cadre de cette stimulation théorique, voir Chantraine et Kaminski, 2007. 
contraire de la normalisation: elle désigne la doctrine sur laquelle s'appuie depuis des siècles la légitimité des conditions défavorables faites aux détenus: la prison, pour être à la fois dissuasive et juste, devrait fournir à ses occupants des conditions de vie nécessairement moins favorables qu'aux plus pauvres des citoyens libres. Depuis que ces trois notions politiques ont été définies, promues ou combattues, le monde a changé. Quelles sont encore leur signification et leur pertinence contemporaines? Comment s'articulent-elles aujourd'hui? D'une façon simple et provocante, je pose la question suivante: si les droits des détenus ne sont que les instruments d'un choix politique favorable à la normalisation, en quoi le projet normalisateur se démarque-t-il encore aujourd'bui de l'option de la moindre éligibilité? La porosité des murs de la prison, présentée souvent comme condition de la détotalisation de l'enfermement, ne signe pas la fin heureuse de l'histoire. Pour preuve:

Lordre, c'est le principe selon lequel tout ne se vaut pas. C'est le contraire de ce système de pensée et de valeurs dans lequel l'élève est l'égal du professeur, dans lequel le délinquant compte autant que la victime, dans lequel celui qui ne se donne aucun mal est autant considéré que celui qui s'en donne beaucoup. [...] L'ordre, c'est quand la loi est la même pour tous, quand le crime est puni et le mérite récompensé ${ }^{4}$.

\section{Droits des détenus}

Il faut rendre la question des droits des détenus à la fois plus sérieuse et à la fois moins juridique. Les droits subjectifs sont depuis deux siècles entourés d'une aura de lutte, de conquête et de victoire, et cela est juste et correct du point de vue de l'action, du militantisme et des bénéfices engrangés. Cette représentation me paraît en même temps fausse au regard de la confiance qu'elle accorde à la performance des droits dans le cadre des transformations de la société post-industrielle.

Les droits de l'homme, historiquement ancrés dans la pénalité, pour en limiter les excès sur le plan normatif, sanctionnateur et procédural (Ost, 2007: 8), doivent se redoubler de nouveaux droits spécifiques ou spécifiquement reconnus, dès lors que, passé à travers ces protections, un individu se retrouve (sans protection) derrière les barreaux, légitimement et légalement, dans le plus splendide respect de ses droits. La

4. Discours du 11 février 2007, prononcé à Paris, par N. Sarkozy, candidat à la présidence de la République française. 
naissance et le développement d'un mouvement visant la reconnaissance de droits aux détenus sont incontestablement liés au mouvement des droits de l'homme. Mais l'ambiguiité est immédiate. Si l'emprisonnement apparaît lui-même comme une victoire humaniste sur les châtiments corporels, sur la peine de mort et sur l'inégalité de traitement, aucun châtiment n'a semblé plus égalitaire et moins inhumain que la privation du temps, de l'espace et du mouvement. Cette victoire historique a montré ses limites et la prolongation de la lutte devait s'opérer à l'intérieur des murs 5 .

Bien plus tard, la promotion politique d'une fonction de resocialisation de la peine était plus à même (jusqu'à un certain point) de soutenir une conception de l'enfermement préservatrice des droits du citoyen détenu. En 1955, les règles minima pour le traitement des détenus (ONU, Genève) préconisent trois principes directeurs (article 56) à travers lesquels des droits se profilent: appartenance des détenus à la société; reconnaissance de la privation de liberté comme châtiment suffisant (dans la filiation de Paterson); et privilège aux moyens «curatifs» et individualisés de la protection de la société contre le crime. On sait que, dans les années 1970, c'est la critique des abus de la philosophie du traitement qui a le plus contribué au retour, aux États-Unis, d'un rétributivisme compatible avec la préservation des droits et justifiés par ces derniers. Et pourtant, ces principes ne sont pas respectés: conditions de vie, travail, statut social, soins de santé ont été, sont et seront encore, dans de nombreux États occidentaux et riches, totalement déficitaires.

Cette première évocation de la malléabilité des droits devant la variation des objectifs pénologiques peut être développée encore. Selon Dupréel, célèbre administrateur belge des prisons, qui ose une conférence en 1958 sur «une notion nouvelle: les droits des détenus», c'est au nom de l'ordre public et singulièrement de la sécurité que des limites légitimes, voire légales, sont placées, de nature à réduire la portée des droits ${ }^{6}$. Le discours naissant dans les années qui ont suivi la fin de

5. Il est intéressant de constater que les expériences d'«enfermement injuste» sont à la base des réformes les plus substantielles des administrations pénitentiaires. Pour ne citer que des Belges, Ducpétiaux (au XIx siècle) et Cornil ( $\mathrm{au} \mathrm{Xx}^{\mathrm{e}}$ siècle) ont connu les prisons des régimes politiques (respectivement hollandais et allemand) dont ils ont été victimes. Voir Maes, 2009.

6. Fonctionne à plein l'illusion qu'il n'y a là rien d'autre que la limitation légitime des droits telle qu'elle est placée aussi pour les citoyens libres. 
la Seconde Guerre mondiale n'hésite pas non plus à faire des droits de l'homme détenu l'instrument de la resocialisation du criminel, tordant doublement la logique: la soumission des droits au service d'un objectif institutionnel et la substitution des sujets (détenu et criminel). Ainsi Dupréel (1957-1958: 168) pense «qu'il est souhaitable de reconnaître aux détenus les droits susceptibles de les aider dans leurs efforts de redressement et de préparation de leur retour à une vie sociale normale». On entend bien la vocation ambiguë des droits, ici au service du redressement (le mot «normalisation» étant synonyme de perspective correctionnaliste). Il continue de façon plus précise encore: «l'octroi de droits non indispensables à des personnes, dont beaucoup sont arrivées en prison par manque de sens social, risque de développer chez elles un sentiment de suffisance, une tendance à utiliser ces prérogatives pour résister aux décisions prises à leur égard dans le cadre du traitement pénitentiaire instauré en leur faveur» (ibid.). On découvre on ne peut plus nettement ici que le droit ne s'accorde guère à la bienfaisance, voire à la générosité. Le bien voulu au détenu en l'occurrence limite sensiblement les droits que, parcimonieusement, on s'apprête à lui reconnaître... On pourra lire à l'envers et sans cynisme ce dernier propos: vouloir le bien de quelqu'un et le reconnaître comme sujet de droit sont deux projets passablement antinomiques.

Vingt ans plus tard, ce n'est plus un administrateur belge qui reprend la thématique, mais bien Pierre Landreville à la fois dans la Revue de droit pénal et de criminologie (1978) et dans la revue Criminologie ellemême (1976). Rien que pour saluer Pierre, j'aurais choisi ce thème aujourd'hui, au risque pourtant de le mécontenter. Le discours du professeur engagé est différent: c'est maintenant le «regain d'intérêt pour les droits des minorités» et le développement de luttes tant internes qu'externes, tant violentes que militantes, qui justifient l'émergence du thème. Le principe de la reconnaissance des droits est nettement affirmé et la question des limites admissibles est radicalement discutée: les détenus conservent tous leurs droits de citoyens sauf ceux qui leur sont expressément ou nécessairement retirés par la loi. Pierre Landreville (1978: 390) soutient que les droits n'ont pas à être limités par une quelconque noble fonction dévolue à l'emprisonnement, fût-ce la réhabilitation: «s'il s'agit de droits, les détenus n'ont pas à prouver qu'ils les méritent ou qu'ils peuvent en bénéficier». Seul l'objectif incontestable de la prison (la restriction temporaire des mouvements d'individus considérés comme dangereux) peut entraîner la restriction des droits. 
Le paternalisme de Dupréel est rejeté, à une époque où les abus de la réhabilitation constituent un des principaux points de focalisation des militants des droits. La valeur soutenue est la réduction des «effets secondaires de la condamnation pénale, de l'incarcération et de la stigmatisation» (Landreville, 1978: 399). Les droits deviennent radicalement forces d'opposition.

Guy Lemire a suggéré, en 1991, un paradoxe inquiétant: «malgré un plus grand respect des droits des détenus, il n'a jamais été aussi difficile de "faire du temps" que dans les établissements dorés actuels» (Lemire, 1991 : 66; voir aussi Lemire, 1990). Un paradoxe proche est proposé par C. Gearty (1991: 221) qui indique, la même année, pour l'Angleterre, qu'après dix ans d'interventionnisme judiciaire en matière de droits des détenus, les rapports tant du Board of Visitors que de l'Inspection sont unanimes pour qualifier les conditions d'incarcération d'intolérables, d'humiliantes, ou de dégoûtantes. On peut d'une part s'indigner avec ces auteurs, d'autre part s'interroger, comme je l'ai déjà fait dans un ouvrage publié en 2002, sur l'apparente identité qu'ils «voudraient» normativement établir entre respect des droits et confort de la détention (Kaminski, 2002: 91; voir aussi Rostaing, 2007). Je voudrais repartir des allégations de Guy Lemire pour aller plus loin. Je le cite: «si on ne peut nier que le discours des droits des détenus ait produit certains résultats positifs, son utilité a finalement été plutôt réduite et, sous certains aspects, il y a lieu de parler d'effets contreproductifs. Le discours ne semble pas en mesure de jouer un rôle déterminant dans la vie quotidienne de la prison» (Lemire, 1991: 75). Si j'accepte volontiers cette analyse, je n'en accepte pas la conclusion: «Vingt ans de discours abolitionniste, d'“alternatives" à l'emprisonnement et de droits des détenus (puisque finalement tout cela se tient) ont mené à des prisons plus surpeuplées et toujours aussi conflictuelles, sans véritable vocation, où domine le plus souvent l'entreposage humain. Le temps est venu de demander des comptes. Nothing works! On a été moins patient avec la rééducation» (Lemire, 1991: 75). L'enjeu de cette évocation des mots de Guy Lemire et de l'amicale dispute que je lui propose est de ne se tromper ni d'analyse ni d'ennemi. Il est probable que la séquence temporelle soit «relativement» juste - les droits des détenus ont accru leur performance et en même temps il n'est pas aujourd'hui plus facile de «faire son temps»-, mais je soutiendrai ici que la séquence causale est incorrecte: le discours abolitionniste et celui des droits des détenus n'ont pas mené à des prisons plus surpeuplées (par 
exemple). Le discours des droits n'est pas la cause de la surpopulation et l'exemple même du sort de la rééducation exige plus que jamais d'être patient. À indexer droit et confort, on s'aveugle sur la véritable nature des droits. La vocation des droits est d'ordre politique; elle n'est pas de rendre la vie plus facile.

La Belgique reconnaît enfin des droits aux détenus, dans une loi votée en 2005 dont l'application se fait attendre. Singulièrement, la couleur radicale des propos tenus dans les années 1970 est fortement présente, matinée cependant d'un nouvel avatar de la justification de la peine de prison, j'ai nommé la réparation, qui change tout. Sous cet avatar, la malléabilité des droits suscite de nouvelles inquiétudes.

Il semble bien que le droit subjectif, dès lors qu'il concerne l'institution ne peut guère s'épanouir, fût-ce discursivement, qu'en se soumettant ou en s'arrimant à l'objectif pénologique et se présenter dès lors comme l'instrument institutionnel d'une politique, autant que l'instrument de défense de la dignité de l'homme contre l'institution. Cette dualité du «droit emprisonné» (Brossat, 2001; Reynaert, 2004) semble structurellement indéfectible et signe un conflit qui n'est jamais gagné par le droit comme tel, dans une pureté, une asepsie que ne soutient encore qu'un positivisme juridique... stérile.

Les évolutions tant sociales que pénales semblent remettre, dans de nombreux États occidentaux, le droit pénal et la prison en position de remèdes évidents et non plus ultimes, vertueux plus qu'odieux (Ost, 2007: 14-15) au mal de l'insécurité. La jurisprudence de la Cour européenne des droits de l'homme est, de ce point de vue, étonnante dans sa façon de soutenir, au détour de certains arrêts, que la mise en œuvre du droit pénal est nécessaire à la protection des droits de 1'homme (Tulkens et van de Kerchove, 2005; Van Drooghenbroeck, 2007). On peut faire l'hypothèse que la dépolitisation de la question des droits de la personne et son revers - la montée en puissance de l'individualisme des droits (Poncela et Lascoumes, 1998: 286-287), favorisé par celle de la victime - rend d'autant plus nécessaire que vienne s'inscrire la lutte pour les droits en prison.

Deux arguments émergent à ce stade de mon propos. Le premier concerne les limites dans lesquelles le droit pénal moderne institue la protection $\mathrm{du}$ «criminel». Le droit pénal classique se caractérise avec bonheur par son garantisme; il en arrête cependant les effets protecteurs au jugement. Il ne prolonge pas le garantisme dû au prévenu (et moins au condamné) jusqu'au cœur de l'exécution de la peine, trou noir de la 
galaxie pénale. Et si les droits de l'homme servent aujourd'hui l'exercice de la pénalité plutôt qu'ils ne le contraignent, une extension de la protection du détenu sur la phase de l'exécution de la peine n'en est que plus nécessaire. Le second argument introduit le doute. L'usage contemporain des droits de la personne au profit de la mise en œuvre du droit pénal pourrait bien servir de leçon pour un examen inquiet de la situation carcérale: et si les droits du détenu se révélaient réversibles, dans l'individualisme qui les caractérise et dans la responsabilisation qu'ils induisent ou pire encore dans leur soumission au projet pénologique de la prison? Je voudrais répéter et surtout compléter ici la thèse que je formulais en 2002:

Loin de dénier les avancées considérables de la reconnaissance de droits substantiels ou procéduraux aux détenus, je pense que cette reconnaissance trouve son expression lorsqu'elle est susceptible de produire des effets fonctionnels et symboliques plus rentables pour le système étatique (en l'occurrence pour son administration pénitentiaire) que les modalités antérieures, disciplinaires et arbitraires, de la régulation carcérale. La crainte qui sous-tend mon analyse est que ce qui peut s'interpréter comme une victoire pour des catégories d'individus démunis consiste plus fondamentalement en un progrès du cynisme managérial, sous l'empire duquel l'État distribue mollement ses responsabilités, exprimées en termes de droits individuels. (Kaminski, 2002: 91)

Gilles Chantraine a formulé mieux que moi le socle théorique de ma critique - redevable à Michel Foucault - en rappelant la distinction entre discipline et gouvernement. Si la discipline met l'accent sur les techniques de surveillance, d'individuation et de normalisation, dans le gouvernement, s'exerce un pouvoir de type «inciter, susciter, combiner» (Chantraine, 2004). Dans le passage contemporain ou postmoderne de la discipline au gouvernement et dans l'association complexe des deux rationalités (voir Cliquennois, 2007), l'accent n'est «plus mis sur les dimensions strictement sécuritaires et répressives, mais plutôt sur le "dispositif" dans lequel elles prennent forme et la diversité des techniques de gouvernement avec lesquels elles viennent s'entrelacer» (Chantraine, 2004). Les droits des détenus m'apparaissent comme des outils d'un progrès significatif, mais conceptuellement dépassé: s'ils sont un outil contre la discipline, ils collaborent au gouvernement. «Les détenus sont ainsi, pour parler comme N. Rose, gouvernés à travers leur liberté [...], cette liberté venant renforcer et complexifier les sanctions traditionnelles et les pouvoirs plus strictement disciplinaires, faisant apparaître une 
technique de gouvernement complexe [...]» (Chantraine, 2004). Que les détenus soient gouvernés à travers leur liberté peut sembler une formule cynique, pourtant son emploi coïncide avec la promotion très protectrice et très idéaliste à la fois du principe selon lequel l'emprisonnement ne doit porter atteinte qu'à la liberté d'aller et de venir. Formule cynique, pourtant compatible avec la logique des droits.

Pour le dire trop simplement peut-être, le bénéfice attendu de l'introduction d'une logique des droits en prison s'amoindrit si l'on constate que la prison est passée d'un seul coup de l'institution totale à l'institution responsabilisante ou sociale-active, et du non-droit au droit mou. Les droits restent un outil de résistance indispensable ${ }^{7}$, mais ils s'intègrent aussi parfaitement dans la nouvelle technique gouvernementale de la prison, importée du «reste du monde», dans un raccourci saisissant, en passant du régime (paternaliste/pervers) de faveurs au régime des droits conditionnels. Le raccourci est saisissant en ce sens que la victoire des droits s'opère au moment même où le droit, dans «le reste du monde», se mue en dispositif de discrimination de ses bons et de ses mauvais usages, de ses sujets méritants et de ses mauvais sujets, en suivant l'intrication des rationalités disciplinaire et gouvernementale.

Plus encore sans doute, lorsque l'on vit de près les conditions régulières, légales, respectueuses des droits, de la détention, l'on se rend compte du piège même de cette régularité, de cette légalité, de ce respect. Le récit de Philippe Landenne (2008) révèle les effets dramatiques de ce qu'il appelle pudiquement l'addition cachée, soit le surplus de souffrances qui, au cœur de la légalité, tue le droit et met fin à la vie. En prison, la vie se joue sur une décision exécutable immédiatement, sur des appels à l'aide jamais entendus, sur des coups de tête parfaitement réciproques des détenus et de l'administration, dans un espace que le droit, avec sa machette émoussée, ne peut défricher, et ne peut déchiffrer avec ses concepts aseptisés, et dans un rythme vertigineux (un «temps réel») avec lequel la lenteur de la procédure administrative ou judiciaire ne peut que rarement rivaliser.

7. La jurisprudence de la Cour européenne des droits de l'homme est significative à cet égard (voir Tulkens, 2002). Le travail remarquable des experts du CPT l'est tout autant. On ne souligne probablement pas assez la ténacité qu'il faut aux requérants devant la Cour pour soutenir leurs droits après des années. Et on ne souligne pas assez l'absence de conséquences générales tirées par les États de leurs condamnations particulières, en raison probablement du premier défaut mentionné ci-dessus. Voir Landenne (2008). 
Margarida Garcia, écrit, pour expliquer la faible performance innovatrice des droits de la personne face au système de pensée du droit criminel, en se soutenant de Morgan (1999), que des organisations - ou des systèmes - se reproduisent de façon redondante lorsqu'elles sont égocentriques, c'est-à-dire lorsqu'elles «ont une idée assez arrêtée de ce qu'elles sont ou, encore, de ce qu'elles pourraient être, et qui sont bien décidées à imposer ou à conserver cette identité à tout prix. Cela les conduit à magnifier leur propre importance, tout en minimisant l'importance et la signification du réseau de relations plus vaste dans lequel elles s'inscrivent» (Garcia, 2007). Et «le résultat de cet égocentrisme, c'est que finalement, beaucoup d'organisations essaient de conserver des identités qui manquent de réalisme ou d'en produire qui, en fin de compte, détruisent d'importants éléments des contextes dont elles font partie» (Garcia, 2007). L'idée est intéressante, mais je crois au contraire que, appliquée à la prison, cette hypothèse ne convient pas. Lorganisation carcérale n'arrête jamais d'importer d'importants éléments des contextes dont elle fait partie, pour le pire et le meilleur. La prison, aussi fermée soit-elle, n'est pas égocentrique, comme l'est le système de droit criminel, auquel Margarida Garcia applique judicieusement son raisonnement. Les deux notions majeures que je voudrais affronter maintenant constituent à mon sens les prises apparemment contradictoires que le contexte inflige à la prison, ou encore les deux indices de la porosité, parfois plus inquiétante que bénéfique, de la prison.

\section{Normalisation}

Le concept de normalisation est récent, dans le sens progressiste où je veux l'utiliser ici, car c'est bien un concept progressiste, extrêmement utile, en période normale. Il est probablement l'héritier d'un État-providence dans lequel l'exclusion est pensée comme le repoussoir social le plus fort. C'est aussi le concept qui soutient le plus solidement le développement des droits des détenus. Lorientation idéologique dite «normalisation» est historiquement associée à la promotion d'objectifs pénaux reposant sur le traitement et sur la réhabilitation, mais une version plus radicale se dégage de ces associations, version dans laquelle prévaut la reconnaissance des droits des détenus, quel que soit l'objectif poursuivi par la pénalité. Les «droits des détenus» ont, avec le temps, pris une autonomie à l'égard de toute philosophie et le concept de normalisation en est un excellent témoin. Il faut protéger les droits, les reconnaître, les 
faire respecter, parce qu'ils ont en eux-mêmes une valeur. La dignité humaine apparaît alors dans une espèce de nudité qu'aucune utilité n'a à voiler. Il s'agit de normaliser les conditions la vie en prison, qui, selon ce principe, devraient être, autant que possible, similaires à la vie extra muros, sauf les restrictions inhérentes à la privation de liberté. Admettons la précision et la portée progressiste de la définition suivante: «La normalisation des relations en prison par rapport à celles en liberté comporte la possibilité pour les détenus d'assumer une responsabilité sociale et ainsi de contrer les effets néfastes de l'incarcération» (Dünkel et Snacken, 2005: 38). Le concept renvoie ainsi aux standards de la vie extérieure. King et Morgan évoquent son application en indiquant qu'autant que les ressources le permettent et dans le respect des contraintes d'un enfermement sûr, exige le respect des mêmes standards qui gouvernent la vie des délinquants dans la communauté (King et Morgan, 1980: 37-40; c'est moi qui souligne): ceci concerne le logement, la nourriture, l'habillement, la santé, l'aide sociale et l'éducation, l'emploi mais aussi le maintien des liens familiaux et sociaux, le traitement des infractions disciplinaires (Snacken, 2001 : 50-51).

Cependant, ce concept invite à une triple prudence. Il contient avant tout sa part de construction de la normalisation de la prison elle-même, j'entends ici de sa considération comme normale. Une normalisation culturelle de la prison et des processus qui y conduisent rivalise avec l'enjeu progressiste de la normalisation des conditions de vie en prison. Ainsi, si la Convention et la Cour européenne des droits de l'homme forment des barrages relativement efficaces contre la peine de mort, contre la torture et les traitements inhumains et dégradants, elles ne permettent que très médiocrement de soutenir que l'emprisonnement constitue un tel traitement. Françoise Tulkens, préfaçant l'ouvrage de Philippe Landenne (2008), interroge les critères contemporains des traitements inhumains et dégradants: "quelles sont aujourd'hui les "exigences légitimes" de la peine? Toute la question est là car ce sont ces exigences que nous devons sans cesse interroger et réinterroger. Tout comme le droit à la vie refuse aujourd'hui la peine de mort, je pense que le droit à la liberté refusera un jour l'enfermement comme peine» (in Landenne, 2008: 9-10).

Le concept de normalisation demande ensuite la définition d'un étalon, «norme» de la vie à l'extérieur, et l'opérationnalisation la plus restrictive possible des limitations «inhérentes» à la privation de liberté. La normalisation, concept promu comme critère d'exigence des droits 
des détenus a fait l'objet de récentes modifications normatives qui tentent de réduire - mais indiquent tout autant - son extrême dépendance à un étalon de plus en plus flou. Pour autant que les ressources le permettent, dans le respect des contraintes de sécurité (formules stéréotypées des restrictions conventionnelles au respect des droits), la vie en prison doit être la plus normale possible. Les auteurs des règles pénitentiaires européennes ont senti le vent de la normalité tourner. Ainsi la règle 5 énonce que: La vie en prison est alignée aussi étroitement que possible sur les aspects positifs de la vie à l'extérieur de la prison. L'introduction du qualificatif «positifs» est commentée de la manière suivante, prenant acte que la vie à l'extérieur de la prison n'était pas ou plus nécessairement aussi «normale» qu' «avant»: il y a lieu de «s'assurer que cette normalisation ne puisse pas avoir pour conséquence des conditions de détention inhumaines ${ }^{8} »$.

L'inscription dans la définition même de la normalisation de sa composante de responsabilisation sociale du détenu soulève enfin un troisième motif de prudence. Pour en avoir éprouvé les effets dans une ample recherche (de Coninck et al., 2005), la valorisation de la responsabilité sociale du justiciable peut s'avérer plus souvent qu'à son tour un boulet bien pire que le constat de sa dépendance objective: ce que nous avons observé dans cette recherche et que Philippe Mary rappelle, c'est que les premiers acteurs responsabilisés aujourd'hui sont les travailleurs du pénal, dans un contexte de surveillance extrême de l'opinion publique et des médias. «La généralisation de la responsabilisation [des acteurs professionnels] s'accompagne d'une généralisation de l'incertitude des acteurs qui tenteront de réduire celle-ci essentiellement par le renvoi en cascade de la responsabilité et de la décision et, in fine, le transfert de la responsabilité sur le justiciable lui-même» (Mary et al., 2006: 399). Les conditions renouvelées de la libération conditionnelle et l'implantation d'une inflexion réparatrice dans la culture pénitentiaire (en Belgique) témoignent de cette perversion de la responsabilité que

8. Sonja Snacken opère une distinction entre normalisation au niveau individuel et normalisation au niveau collectif. Au niveau individuel, la normalisation signifie la reconnaissance de la diversité des rôles sociaux du détenu (diversité niée par l'institution totale), l'accroissement de la responsabilité et de la place accordée aux aspects positifs de sa personne (Snacken, 2002: 138). Au niveau collectif, le principe de normalisation signifie que «les services collectifs octroyés en prison doivent être autant que possible équivalents à ceux offerts à l'extérieur» (Snacken, 2002: 145): le travail et les soins de santé en prison sont les deux exemples les plus significatifs en la matière. 
la normalisation voudrait pourtant soutenir à des fins de transformation réelle de la prison?.

Mon projet de désincarcération et de désidéalisation de la question suppose d'interroger la fragilité du concept de normalisation. Cette fragilité est celle-là même qui affecte d'autres concepts relatifs, comme le concept de modération ${ }^{10}$.

Dans la vie libre ou «normale», de plus en plus de gens se voient nier la diversité de leurs rôles sociaux ainsi que les aspects positifs de leur personne. Dans la vie libre, les services collectifs octroyés ne font que s'amenuiser. Dans la vie libre enfin, est sérieusement remise en cause la «positivité» de certains aspects de la vie qui étaient évidemment positifs dans la période que Richard Sennett qualifie de «capitalisme social» (2008). La vie libre contient de plus en plus de conditions inhumaines. La normalisation des conditions de vie en prison trouve étonnamment sa reconnaissance symbolique au moment où s'effrite, hors de la prison, l'étalonnage de la vie normale. Devant l'errance normalisée des itinérants, devant le sort infect mais normalisé des demandeurs d'asile, devant la reconnaissance normalisée que, dans mon pays, $15 \%$ des habitants vivent sous le seuil «officiel» de pauvreté, devant la

9. Il faut cependant ne pas confondre la victoire symbolique du concept et les constats empiriques qui sont déplorables à tous niveaux. En ce qui concerne le travail, les constats de Johannes Feest (1999) sont reconduits par Evelyn Shea (2005). Quant aux soins de santé, parmi de nombreuses publications, je n'évoquerai que celle de Rhidian A. Hughes (2000): les besoins des détenus couvrent un spectre très étendu et ne sont pourtant que rarement satisfaits. La discussion de l'auteur tourne autour d'un enjeu sémantique significatif: pour développer une politique de normalisation de l'accès et du droit aux soins, faut-il continuer à nommer le détenu par cette appellation ou l'appeler patient?

10. Comme l'écrit Pierrette Poncela: «La modération des peines fut l'un des premiers droits de l'homme revendiqué et proclamé; il fut toujours et demeure l'un des plus incertains, l'un des plus sujets à variation» (Poncela, 2007: 376) associé historiquement à celui de «nécessité des peines» puis de proportionnalité. Mais la modération est elle-même un concept bien mou. Mon propos veut surtout insister sur le redoublement de cette mollesse conceptuelle relative au sentencing, lorsqu'on se situe à l'intérieur de la prison: la proportionnalité et la modération sont des concepts qui n'ont plus cours, même lorsque la Cour européenne des droits de l'homme a à juger du caractère inhumain et dégradant de la longueur inhabituelle de la peine lorsque le détenu se voit refuser toute perspective de libération conditionnelle (par exemple, l'affaire Léger, évoquée par Pierrette Poncela [2007], du nom d'un détenu français admissible à la libération conditionnelle depuis 1979 et l'ayant obtenue en 2005). Plus encore, que serait la modération de l'exécution de la peine, entendue comme condition de la vie carcérale? C'est probablement à cet endroit précis qu'interviennent les droits du détenu, mais comment croire à leur performance sensible intra muros si l'on convient qu'en amont le même type de critère ne peut déjouer les processus de pénalisation croissante dans laquelle le principe de proportionnalité est «bousculé» comme le démontrent Christine Guillain et Damien Vandermeersch (2007: 414). 
croissance fulgurante de l'emploi précaire et temporaire et pourtant normalisé, devant la dérégulation normalisée des salaires et des horaires de travail, devant la religiosité compassionnelle encourageant la sanctification temporaire des victimes du moindre crime crapuleux, devant la triple exigence capitaliste du non-engagement à long terme, de la valorisation du potentiel au détriment des compétences acquises et de la négation du passé (Sennett, 2008), devant la normalisation de l'indignité, qu'est-ce que la normalisation des conditions de vie en prison?

La normalisation en prison apparaît aujourd'hui comme un processus retardataire, courant après un standard improbable. Outre le fait qu'elle ne peut que choquer la construction politique de l'opinion publique, elle ne peut que s'essouffler derrière un étalon enfui. Si le droit n'est que l'instrument d'une politique, d'un conflit qui n'est jamais gagné par le droit comme tel, alors la promotion des droits pour euxmêmes est un leurre idéologique qui me paraît avoir pour effet paradoxal de les laisser flotter et se mettre au service des rationalités nouvelles qui, aujourd'hui, en infléchissent le tranchant. Le vent politique de la normalisation a perdu de son piquant. Le développement des droits conditionnels ou des droits comme instruments d'activation, tellement violent déjà dans les politiques sociales, constitue la matrice d'une relecture contemporaine de la reconnaissance des droits du détenu, lecture dans laquelle peu de choses séparent encore normalisation et moindre éligibilité.

\section{Moindre éligibilité}

Lorientation idéologique dite de la «moindre éligibilité ${ }^{11}$ », la plus constante et la plus ancrée dans l'évidence, dévalue la citoyenneté des détenus, en raison de leurs crimes, et les soumet à des conditions de vie dans lesquelles peu de droits méritent de leur être concédés.

Si l'on s'en tient à la période moderne, creuset de ce qui nous reste de démocratie, le principe de la moindre éligibilité est formulé en France par les procès-verbaux du Comité de mendicité de la Constituante, crée en $1790^{12}$. Thomas Malthus publie en 1798 son Essai sur le principe

11. Johannes Feest (1999: 100) indique que la normalisation et la moindre éligibilité sont en fait les noms de deux orientations idéologiques contrastées qui permettent de nouer des éléments disparates de la description des conditions de vie en prison.

12. Le comité de mendicité écrit: «L'homme secouru par la Nation et qui est à sa charge doit cependant se trouver dans une condition moins bonne que s'il n'avait pas besoin de 
de population, d'entrée de jeu consacré à un problème qui n'est pas que pénitentiaire: la surpopulation menaçante. Marjie Bloy (s. d.) rappelle qu'une des causes de la surpopulation est notamment politique: la fourniture d'une assistance aux pauvres mesurée au nombre d'enfants. S'introduit ici l'idée que cette «mesure» encourageait les pauvres gens à faire de nombreux enfants pour accroître le volume d'aide alloué. Ce rappel historique de la doctrine de la moindre éligibilité témoigne de l'accointance de deux logiques, l'une morale et l'autre économique, l'une individuelle et l'autre macro-sociale, s'alliant pour favoriser, dans les politiques sociales, une discrimination bien mesurée destinée à éviter les effets pervers que produisent des hommes rationnels, à moins que ce soient les effets rationnels que produisent des hommes pervers.

La formule appartient à Charles Dickens ${ }^{13}$ : il est monstrueux que le pauvre malhonnête soit mieux traité que le pauvre honnête. Je le dis malgré tout le bonheur que je retire encore de la lecture de Dickens: cette formule est elle-même monstrueuse. En effet, elle n'ouvre pas l'option de l'amélioration des conditions de vie des pauvres, mais bien la seule option du durcissement des conditions de vie institutionnelles des pauvres qui ont démérité. Il est intéressant de constater à quel point la discrimination des pauvres en méritants et non méritants est au cœur des discours électoraux du candidat Sarkozy à la présidence de la République française (quelqu'un m'a dit qu'il a fini par être élu). La méritocratie est un concept-clé pour comprendre la reconfiguration de la question sociale dans le capitalisme contemporain (dont un Sarkozy est à la fois le chantre et le bouffon) et l'inflexion qu'y prend la notion de droit subjectif.

Ce principe économico-moral naît donc dans le champ de l'assistance, puis se répand dans celui de l'enfermement des pauvres, enfin dans le champ péna $1^{14}$. Une dimension punitive rétributive est d'entrée de jeu présente dans le principe, où qu'il s'applique; il ne pouvait

secours, et qu'il pût exister par ses propres ressources; le besoin qui naît du manque de travail dans un homme qui n'en a pas cherché, dans celui qui n'a pas pensé à s'en procurer, pénible sans doute pour un cœur humain et compatissant, est, dans un État où il y a une grande masse de travail en activité, une punition utile et d'un exemple salutaire» (cité par Keslassy, 2000: 330).

13. Dans $A$ Walk in a Workhouse (1850): «we have come to this absurd, this dangerous, this monstrous pass, that the dishonest felon is, in respect of cleanliness, order, diet, and accommodation, better provided for, and taken care of, than the honest pauper».

14. G. Rusche et O. Kirchheimer (1939; éd. française, 1994) en commentent les effets dans leur célèbre ouvrage. 
qu'avoir vocation à s'appliquer particulièrement en prison. N'est-il pas évident et sain que les conditions de détention soient moins favorables que les conditions les plus élémentaires de la vie libre? Sans quoi les pauvres hommes rationnels viendraient supplier aux portes des prisons qu'on les laisse entrer pour bénéficier du régime plus favorable qu'on y vit. Ce principe, dans l'esprit de l'État-providence s'éclipse au $\mathrm{xx}^{\mathrm{e}}$ siècle, dans une période bénie, mais souvent présentée comme perverse aujourd'hui, où l'option politique privilégiée consistait, non plus à assurer la survie des pauvres, mais à égaliser la société et à relever les conditions élémentaires de la vie. La moindre éligibilité ne disparaît pas aussi nettement qu'ailleurs dans le champ pénitentiaire, tant le comportement coupable du délinquant (et non seulement le comportement «paresseux» de l'assisté) légitime la discrimination et tant persiste la représentation religieuse d'une punition qui doit représenter l'enfer sur terre.

Il serait facile de se contenter dans cette communication de faire valoir que la moindre éligibilité aurait aujourd'hui la cote et surmonterait son challenger «normalisation». Il serait ainsi facile (quoique!) de montrer par exemple que la privatisation des établissements pénitentiaires (Blakely et Bumphus, 2004) constitue une réponse au souci de réduire les coûts de l'emprisonnement, voire d'en tirer des profits, et qu'à cet égard, on peut penser que la doctrine de la moindre éligibilité y rend un service appréciable. Il serait facile aussi de montrer que la reconnaissance de certains principes éminemment appréciables contient son venin de moindre éligibilité ${ }^{15}$. Mon propos sera plus grave: je voudrais plutôt attirer l'attention sur les glissements sociaux contemporains qui émoussent l'opposition entre normalisation et moindre éligibilité.

L'État-providence se retrouve responsable et débiteur des effets des luttes gagnées pour les droits des «prolétaires» (par exemple), des «femmes» ou des disqualifiés de toutes sortes (voir Bauman, 1994; Macquet, 2003). La reconnaissance des droits des minorités, corrélative de leur minorisation, représente une victoire incontestable. Mais pendant que cette victoire a lieu sur des terrains multiples et parcellaires (et non à cause de cette victoire), c'est tout le régime des droits qui se

15. Par exemple, l'article 93 des règles minima pour la protection des mineurs privés de liberté de 1990, dites «Règles de La Havane», énonce que L'enfant qui reste avec ses parents détenus doit être l'objet de ménagements et de soins spéciaux, car cet enfant n’a commis aucun crime ni délit. 
modifie et s'infléchit sensiblement. Les droits-libertés se réduisent sous l'effet de politiques antiterroristes ou de politiques sécuritaires contre les incivilités (voir Mary, 2006) et les droits-créances s'infléchissent à la mesure de l'imposition d'un «droit d'accès» à leur bénéfice, quand les caisses se vident. La créance existe, mais elle n'est exigible que sous condition d'un mérite particulier, qui sera singulièrement scruté. L'exercice de nombreux droits est, dans la vie libre, insensiblement devenu conditionnel, autrement dit soumis à la vérification et à l'exigibilité d'un mérite.

L'État-providence, distributeur de droits sociaux jusque dans la prison normalisée, est en effet attaqué de toutes parts : selon une lecture du comportement comme choix rationnel, c'est-à-dire une lecture qui considère a priori le sujet comme pervers, le revenu de l'emploi serait devenu trop peu attractif au regard des revenus de remplacement attribués par l'aide sociale ou le chômage. La formule magique de l'activation (même si son nom n'apparaît pas explicitement) a vite essaimé en dehors des politiques sociales pour devenir le credo "incontestable» de toutes les formes de prise en charge de populations marginalisées ou défaillantes. Autant l'activation renvoie à des valeurs significatives et positives de responsabilisation, de prise en charge de soi-même, d'autonomie, autant elle contient sa perversion anti-démocratique ${ }^{16}$ et inégalitaire. Dans le champ pénitentiaire, l'activation s'inscrit parfaitement dans la perspective d'une réinsertion sociale dont l'acteur principal serait le détenu lui-même à travers le développement d'outils comme le plan de détention ou le plan de réinsertion. Ces outils sont des formes quasi-contractuelles de co-définition des conditions de vie en prison et des conditions individualisées de l'éventuelle sortie anticipée du détenu. La normalisation des conditions de vie en prison peut passer par ce régime d'activation. Mais à mon sens, elle est contaminée et pervertie par l'importation, dans le chef du sujet détenu, de ce nouveau régime de responsabilité que l'État social actif fait peser sur les sujets. Ces «contrats» sont des monstres juridiques, comme le suggère Alain Supiot

16. Un journal économique et financier belge, nommé L'écho, donne le ton de la nouvelle configuration, dans la publicité qu'il s'est donnée en 2008: "Soyez de ceux qui comptent.» La formule est très précise et heuristique en diable. "Que ceux qui ne comptent pas comptent» est très précisément le challenge démocratique - selon les termes de Jacques Rancière (1995) - et normalisateur, mais la démocratie, c'est la reconnaissance, dans les rapports de force qui continuent d'exister, de la responsabilité collective de ce programme. Aujourd'hui, la responsabilité de compter ou non est renvoyée aux sujets capables ou non d'en être». 
(2000; 2005); ils ne sont pas destinés à l'échange mais bien à l'exercice d'un pouvoir.

Deux conséquences concernent directement la situation des détenus: l'individualisation (sous le couvert du respect du droit) du rapport de force et la contrainte au talent exigé de l'entrepreneur.

\section{Le respect du droit et la force de la transaction individualisée}

L'encellulement individuel est un excellent analyseur. Ducpétiaux, dans le $\mathrm{XIX}^{\mathrm{e}}$ siècle belge en faisait un credo axé sur la fonction rédemptrice de la solitude et sur la corruption de la promiscuité des criminels. La version finalement adoptée de la loi belge de 2005 escamote ce droit, pourtant soutenu dans la proposition de loi (à des fins de normalisation des conditions de détention), jusqu'à ce que la ministre de la Justice de l'époque fasse valoir l'impossibilité économique (restriction classique) de la réalisation de l'encellulement individuel. En France, une loi de 1875 impose l'encellulement individuel des détenus, loi qui aurait dû être rendue applicable à la date du 15 juin 2003. En 2008, Rachida Dati (garde des Sceaux) révèle sur cette question l'extraordinaire inflexion contemporaine de la notion de droit, qui érode aussi bien son indexation au principe de la normalisation que son indexation à tout principe pénologique. Je dois les informations suivantes à Jean Bérard et à Gilles Chantraine (2008: 21 et suiv.). Un décret de juin 2008 affirme que le droit à l'encellulement individuel est respecté. Les prévenus qui sont enfermés dans des cellules collectives peuvent demander le respect de leur droit à l'encellulement individuel et, dans les deux mois, on leur répondra en indiquant la prison (n'importe où en France) dans laquelle ils pourront accéder à une cellule individuelle. Pour rester auprès de leur famille ou pour préparer au mieux leur défense, il est certain que les détenus refuseront leur transfèrement à l'autre bout du pays. Rachida Dati peut ainsi conclure que de nombreux détenus ne souhaitent pas être seuls en cellule. La reconnaissance du droit se fait elle-même perverse: le droit du détenu est singulier et n'entraîne pas une obligation collective pour le chef de l'administration, qui pourra jouer, à partir de chaque demande individuelle, un droit contre un autre, un droit contre un intérêt, et démontrer qu'«il n'est pas certain que les détenus veuillent l'encellulement individuel» (Bérard et Chantraine, 2008: 23). L'exemple montre: 1) que le droit subjectif n'est aujourd'hui considéré que comme une prérogative individuelle sans conséquence en 
termes d'obligations politiques collectives; 2) que le respect du droit se mérite, c'est-à-dire se monnaie et se paie, qu'il est devenu procéduralement l'objet d'une transaction coûteuse et non un levier de normalisation. Je peux encore formuler ces deux aspects d'une autre manière: le droit est opposable à l'administration mais ne doit pas être respecté a priori par elle; le droit devient un levier de revendication si coûteux qu'il est préférable de ne pas s'en prévaloir.

\section{Qui peut le moins peut le plus}

Dans l'État-providence, il est reconnu que le défaut de capacités doit faire l'objet d'un filet de sécurité, donnant au mot droit sa pleine portée de protection. Dans la prison de l'État social actif, la normalisation se marie avec l'exclusion en vertu du renversement du dicton: qui peut le moins peut le plus. Le droit reconnu devient une charge individuelle qu'il faut avoir la capacité sociale de porter, de défendre, montrant à terme qu'on en a mérité le respect. La moindre éligibilité sociale est ainsi inscrite à l'intérieur de la logique contemporaine des droits: la moindre éligibilité est réservée à celui qui ne saura pas saisir les ressources personnelles qu'on lui suppute abstraitement pour être «de ceux qui comptent», de ceux qui auront mérité l'effectuation du droit par l'administration. Celui-là ne comptera pas et il n'aura à s'en prendre qu'à lui-même. Il n'aura pas vraiment voulu être seul dans sa cellule. Voilà la normalité qui s'avance, le régime gouvernemental nouveau de l'État social actif, ce régime d'auto-exclusion par la faute individuelle qui consiste à n'avoir pas su saisir ses propres chances supposées égales. La normalisation des conditions de vie en prison ne peut qu'être un alignement tendanciel sur ce nouveau régime de discrimination par les compétences subjectives considérées comme abstraitement égales chez les sujets de droit que nous sommes tous. Cette compétence - ou plutôt ce «talent» attendu du détenu (comme du cadre de n'importe quelle entreprise) - consiste à devenir expert en processus, «être capable de se projeter dans le futur en imaginant ce que l'on pourrait faire en brisant contexte et référence» (Sennett, 2008: 101). L'exigence d'un tel talent pèse, assez normalement, sur l'homme en position de puissance. En se démocratisant, cette exigence se transforme en principe de sélection: «Il n'est guère de plus solide forme de domination que de considérer le faible comme un puissant» (Kaminski, 2006: 337). C'est le second effet du droit mérité. 
Par ailleurs, tant dans la loi que dans le public, la position de la victime ajoute sa couche proprement pénale et carcérale au retour d'une moindre éligibilité décomplexée. La répression se fait aujourd'hui «plus étendue et intensive, [lorsqu'est considéré] que les "droits de l'homme" des victimes sont atteints» (Henzelin, 2002: 105, cité par Van de Kerchove, 2007: 360-361). Le rôle de la victime, accru dans le procès pénal, s'est aussi accru dans la détention. Cette performance de la porosité de la prison n'est pas assez relevée: la figure de la victime a aussi pénétré en prison, de façon bien plus efficace que les autres figures de soutien ou de resocialisation du détenu. Le détenu est invité à faire de sa détention une expérience réparatrice, de planifier l'usage de ses ressources au regard de sa dette civile. L'objectif de réparation, à l'aune duquel ses sorties et libérations possibles sont dorénavant soumises, donne prise à la victime, au nom de ses droits. La nouvelle minorité à protéger, minorité à laquelle nous nous identifions très facilement tous, est celle-là : c'est ce citoyen mineur qu'est la victime. Dans cette nouvelle configuration des rapports de force entre droits de l'un et droits de l'autre, la situation du détenu fait constamment l'objet d'indignations, adressées en même temps qu'à la justice dans son ensemble: les victimes rappellent à qui veut l'entendre que les privilèges du détenu ne leur sont pas accordés à elles (voir Robert, 2007).

La moindre éligibilité doit donc être désincarcérée si l'on veut comprendre mon propos. Sa carcéralisation empêche de l'inscrire dans un continuum entre vie libre et vie carcérale. La moindre éligibilité est inscrite, dans l'entreprise capitaliste nouvelle ainsi que dans les politiques sociales d'assistance aux fins de dissuader «le travailleur de devenir assisté en lui assurant un meilleur statut, [elle] préserve aussi la croyance en une juste hiérarchie sociale, reposant sur une infériorité sociale contrôlée de ceux qui ne travaillent pas par rapport à ceux qui travaillent. Enfin, en rendant l'assistance relativement inconfortable et stigmatisante, en l'opposant au travail et en opposant l'assisté au travailleur, on divise les classes populaires et on fournit un exutoire facile à la frustration des salariés les plus défavorisés. En faisant de l'assisté et de l'assistance une figure repoussoir et dégradante, on incite ceux qui travaillent à rester sur le marché de l'emploi et à supporter leur sort en acceptant des conditions de travail dégradées» (Trombert, 2008) ${ }^{17}$.

17. Christophe Trombert nous donne à voir la fragilité de la normalisation carcérale, par la fragilisation des normes de l'assistance et du travail extra-muros: «Le durcissement 
La distinction carcéralo-centrée entre le vrai désavantagé (le pauvre travailleur) et celui qui ne mérite pas le respect en raison de son crime est aujourd'hui d'autant plus revivifiée que ce modèle de distinction rétributiviste ${ }^{18}$ est à l'œuvre dans la vie libre entre les multiples espèces de pauvres travailleurs que la société contient: le travailleur intérimaire, le travailleur au noir, le travailleur clandestin, le chômeur, le titulaire d'allocations de survie, le clandestin sans travail, le demandeur d'asile et la travailleuse.

Au moment où la politique sociale devient rétributiviste et où, dans la vie libre, la doctrine de la moindre éligibilité fait fureur pour faire accepter des conditions de travailleur et des conditions d'allocataire dégradées, que veut encore dire la normalisation en prison? Sinon un alignement (seulement tendanciel) sur la dégradation généralisée qui affecte la vie libre. La normalisation en prison ne coûte rien quand la moindre éligibilité et la méritocratie règnent extra muros. Les droits du détenu ne peuvent guère être efficaces, comme instruments de dignité et de sécurité, lorsque bien évidemment ils s'alignent sur la conditionnalité «activante» qui nous vient de l'extérieur. La porosité des murs de la prison prend ici un sens moins optimise que dans sa version habituelle de détotalisation de l'univers carcéra ${ }^{19}$.

Je conclus brutalement: pendant que la normalisation fait son chemin dans le monde pénitentiaire, la moindre éligibilité fait le sien, bien plus vite, dans le reste du monde. Si la privation de liberté change avec le temps, la liberté aussi. La porosité de la prison n'est donc pas un gage d'amélioration de la vie des détenus. L'activation du détenu est une stratégie de maintien, voire de renforcement d'une moindre éligibilité qui fait son retour extra-muros et qui s'avère d'autant plus cruelle intra

des conditions d'assistance n'est que le pendant du durcissement des conditions de travail et de la sélectivité de l'emploi; lorsque le travail et l'emploi deviennent plus difficiles, il est nécessaire de dégrader un peu plus la position de non-travailleur afin d'assurer malgré tout au travailleur qu'il s'en sort relativement mieux que d'autres» (Trombert, 2008).

18. La thématique de l'activation des chômeurs contient en effet, selon les termes de Chr. Trombert (2008), une dimension rétributiviste: elle «exacerbe la dénonciation des fraudes, des abus et de l'assistanat. Tant dans le discours des professionnels de l'insertion que dans le discours politique, on note un durcissement de la suspicion envers les assistés et de la dénonciation des fraudeurs et de ceux qui abusent. Ce discours est marqué de considérations rétributivistes dénonçant l'injustice que représente le fait de toucher» une allocation sans rien faire.

19. J'en voudrais pour preuve les résultats du travail de fin d'études de Fabienne Philippe (2008) qui analyse la vie dans la rue (celle des squatters) et qui conclut de façon convaincante que la rue est une institution totale. 
muros. Pendant que la normalisation avance en prison, la méritocratie avance dans la société. La normalisation s'arrêtera donc, dans le meilleur des cas, à reconduire le principe de la moindre éligibilité. Autrement dit encore, la rhétorique et la pratique des droits, même soutenues par un principe de normalisation, sont suffisamment malléables pour être valorisées sans conséquence à une époque où - les droits étant conditionnalisés dans la vie libre - l'étalon de la normalité est devenu un cheval fou.

\section{Alors quoi?}

En inscrivant la thématique des droits des détenus dans le contexte social contemporain, en montrant l'émoussement de la différence tranchée entre moindre éligibilité et normalisation, voire leur complicité, je ne fais pas une critique des droits. Je ne les jette pas avec l'eau du bain: j'attire l'attention sur l'eau du bain dont la couleur saumâtre contamine le bébé et lui fait perdre sa belle santé. Je fais par contre une critique d'une rhétorique des droits qui les considérerait comme des acquis juridiques et non comme des instruments de lutte à renouveler et à intégrer dans une politique qui contient d'autres instruments, en raison même de la dépendance extrême de leur effectuation et de leur performance au contexte sociopolitique dans lesquels ils s'inscrivent ${ }^{20}$. Mais plus encore, je pense que les droits des détenus ne seront jamais à eux seuls un instrument politique, tant le nouveau capitalisme a réussi à faire percoler ses dispositifs gouvernementaux jusqu'au cœur même de la rationalité répressive de l'État.

Les droits des détenus sont donc indispensables, mais dépassés dans la performance qu'on attendait d'eux et je pense que c'est en raison de cette péremption que leur reconnaissance s'obtient sans trop de difficulté, sous une forme médiocre, aujourd'hui. Il faut donc dépasser ce dépassement. Le désenfermement de la pensée politique sur la prison ne passera-t-il pas par la sortie de la «bouteille à mouches» de la pensée juridique? Si les droits de la personne sont (selon la formule d'Alvaro Pires) devenus un obstacle à la mutation du droit pénal (entendue comme innovation progressiste), irait-on jusqu'à penser que les droits des détenus sont un obstacle à une mutation «réellement civilisatrice» de la prison? Non, les droits sont un outil, mais à certaines

20. Tout l'enjeu de l'article de Dan Kaminski et Gilles Chantraine (2007) tient dans l'exigence d'une lecture sceptique, bien qu'«il ne soit pas indifférent» que des droits soient reconnus et revendiqués. 
conditions non remplies jusqu'ici. Je suggère donc, dans les lignes qui suivent, les conditions d'une politique renouvelée de normalisation de la prison, les conditions du soutien du radicalisme oublié de la promotion des droits, pour que l'utopie soit rappelée contre l'idéalisation. Tendanciellement, il y a lieu de tout faire pour lever les obstacles «culturels» à la pleine effectivité des droits, entendue comme application réflexive du principe de normalisation à l'exercice des droits des détenus : 1) lever l'obstacle sécuritaire, soit lutter contre l'hégémonie de la fonction sécuritaire de la prison et de son statut d'argument pour limiter les droits et leur exercice; 2) lever l'obstacle pénologique, soit la soumission de la logique des droits à l'un ou l'autre objectif de la peine; 3) lever l'obstacle économique, entendu à travers la soumission de l'exercice des droits aux ressources affectées à l'administration pénitentiaire; 4) lever l'obstacle social, soit réduire l'empire néolibéral de la doctrine de la moindre éligibilité. Il n'y a guère de recette, mais je voudrais permettre de penser l'effectuation des droits des détenus, dans leur nature que j'ai définie plus haut comme politique.

Conformément aux propositions faites par trois directeurs de prison belges (de Thier et al., 2005 ; voir aussi Rostaing, 2007) pour assurer la meilleure effectivité des droits des détenus, leur exécution devrait être rapide (une décision préjudiciable et injuste produit des effets irrémédiables immédiats), lisible (comment le droit pénitentiaire serat-il rendu lisible même pour un analphabète?), surreprésentée (les nouveaux droits des détenus ne peuvent masquer la privation de droits essentiels que le fait même de l'incarcération produit : la prison doit être un lieu de sur-droit) et mobile (que le droit et ses agents puissent circuler librement dans la prison à la rencontre des besoins de tous) ${ }^{21}$.

Il faut encore que les droits deviennent des obligations institutionnelles et non des prérogatives individuelles dont les conditions de mobilisation ne sont que difficilement effectives et encore moins efficaces en prison. Il est dommageable que la reconnaissance de droits consiste en la transformation (nécessaire à la gouvernementalité contemporaine) par

21. Voir aussi, à ce sujet, le rapport de la Law and Justice Foundation (2008), Taking justice into custody: research into prisoners' and ex-prisoners' legal needs: "The research identified opportunities for prisoners to access legal information, advice and assistance, while acknowledging that the very nature of the prison environment often compromises access to, and availability of, resources including contact with lawyers. This leads to a high level of dependency on others to complete certain tasks, which can result in delays and at times lost opportunities to address outstanding legal problems.» 
réduction d'un conflit politique (d'enjeux collectifs) en conflits réglés entre parties individualisées (Scheingold, 1974). Autrement dit, il faut que les droits des détenus deviennent un programme de politique pénitentiaire et que la reconnaissance des droits ne soit pas seulement ce don de faveurs individuelles et conditionnelles, dont on sait déjà la fragile performance de toute action toujours en retard dramatique sur la réalité vécue, mais la reconnaissance politique et proactive des conséquences institutionnelles qu'il y a lieu à tirer de ce don. L'institution carcérale, au nom de la logique des droits, doit non pas s'adapter au coup par coup à la témérité juridique de quelques détenus quérulents, mais devenir, de sa propre initiative, dispositif de respect des droits ${ }^{22}$.

Enfin, il y a lieu, comme je l'ai rapidement mentionné ci-dessus, de réduire l'empire néolibéral de la doctrine de la moindre éligibilité. Si les conditions de détention deviennent réellement attirantes pour ceux qui vivent la moindre éligibilité en liberté, il faut que cette indignation soit tournée non contre la privation de liberté, mais contre les conditions dégradées de la vie «normale». Si nous vivons dans des conditions pires que les détenus, faisons de cette jalousie une arme politique pour améliorer les standards déréglés de la vie de tous, au lieu de nous satisfaire d'une vie inchangée, en amoindrissant celle des détenus. Cette piste concerne aussi le rapport tendu entre détenus et surveillants: les conflits sociaux du monde pénitentiaire sont presque systématiquement des conflits qui concernent l'éligibilité respective des détenus et des surveillants et sont destinés à confirmer que la moindre éligibilité doit être accordée au groupe des détenus par rapport à celui des surveillants. Ladministration semble se satisfaire de la récurrence de tels conflits, alors même qu'il me paraît certain que chacun des deux groupes (détenus et surveillants) a tout à gagner de l'accroissement de l'éligibilité de l'autre. Le même raisonnement, oserais-je le dire, vaut pour le rapport tendu à outrance entre les auteurs d'infractions et les victimes.

Les droits des détenus étaient conçus hier et sont encore aujourd'hui une arme contre l'institution bienveillante et malveillante à la fois qu'est la prison disciplinaire et resocialisatrice. Que seront-ils demain ou que

22. Un tel devenir exige une éducation ferme, assortie de sanctions et de stigmatisations inlassables, de rappels à l'ordre exercés par des détenus tenaces et leurs avocats irritants, des intervenants communautaires, des organisations militantes, le Comité (européen) de prévention de la torture, la Cour européenne des droits de l'homme (avec, en son sein, Françoise Tulkens), des professeurs de pénologie (avec, parmi eux, Sonja Snacken) qui forment les futurs administrateurs... autant d'outsiders et d'insiders vigilants. 
sont-ils déjà aujourd'hui face à la prison autonomisante et réparatrice, et dont la malveillance se lit déjà dans les excès de subjectivation et de mobilisation des «talents» qu'elle impose?

Il n'y a pas lieu de bouder le bonheur qui envahit le monde depuis trois jours, mais la question vaut d'être posée: quel visage montrera Barack Obama? Incarnera-t-il une plus grande justice sociale et une moindre discrimination pénale ou reconduira-t-il le cauchemar américain selon lequel tout est possible... à qui le mérite?

\section{Références}

Bauman, Z. (1994). Alone Again. Ethics After Certainty. London: Demos, http:// www.demos.co.uk/publications/aloneagain.

Bérard, J., \& Chantraine, G. (2008). 80000 détenus en 2017? Réforme et dérive de l'institution pénitentiaire. Paris: Ed. Amsterdam.

Blakely, C. R., \& Bumphus, V. W. (2004). Private and Public Sector Prisons. A Comparison of Select Characteristics. Federal Probation, 68 (1), 27-31.

Bloy, M. (s. d.). Effect of the reform on the morals of the poor. National University of Singapore, http://www.victorianweb.org/history/poorlaw/morals.html.

Brossat, A. (2001). Pour en finir avec la prison. Paris: La Fabrique éditions.

Chantraine, G. (2004). Prison et regard sociologique, pour un décentrage de l'analyse critique. Champ pénal, 1, http://champpenal.revues.org/document 39.html.

Chantraine, G., \& Kaminski, D. (2007). La politique des droits en prison. Champ pénal, Innovations Pénales, http://champpenal.revues.org/document2581. html.

Cliquennois, G. (2007). De l'entrelacement des disciplines et des dispositifs de sécurité en prison. In M. Cicchini \& M. Porret (eds.), Les sphères du pénal avec Michel Foucault (211-222). Lausanne: Antipodes.

de Coninck, F., Cartuyvels, Y., Franssen, A., Kaminski, D., Mary, Ph., Rea, A., \& Van Campenhoudt, L. (2005). Aux frontières de la justice, aux marges de la société. Une analyse en groupes d'acteurs et de chercheurs. Gent: Academia Press, Politique scientifique fédérale.

de Thier, F., Lebrun, V., \& Spronck, V. (2005). Murmures d'une prison: cela en vaut-il la peine? In Association syndicale des magistrats, La prison, droit dans le mur? Colloque du Xxv anniversaire, Bruxelles: 28 janvier 2005 (non publié).

Dünkel, F., \& Snacken, S. (2005). Les prisons en Europe. Paris: L'Harmattan.

Dupréel, J. (1957-1958). Une notion nouvelle: les droits des détenus. Revue de droit pénal et de criminologie, 163-173.

Feest, J. (1999). Imprisonment and Prisoners' Work: Normalization or Less Eligibility? Punishment $\mathfrak{V}$ Society, 1, 99-107. 
Garcia, M. (2007). Innovation et obstacles à l'innovation : la réception des droits de la personne par le système de droit criminel. Champ pénal, Innovations pénales, http://champpenal.revues.org/document1192.html.

Gearty, C. (1991). The prison and the courts. In J. Muncie \& R. Sparks (eds.), Imprisonment: European Perspectives (219-242). New York: HarvesterWheatsheaf.

Guillain, Chr., \& Vandermeersch, D. (2007). Les droits de l'homme en droit pénal et en procédure pénale: effectivité ou alibi? In Y. Cartuyvels, H. Dumont, F. Ost, M. van de Kerchove \& S. Van Drooghenbroeck (eds), Les droits de l'homme, bouclier ou épée du droit pénal? (377-426). Bruxelles: Publications des FUSL.

Henzelin, M. (2002). Droit international et droits pénaux étatiques. Le choc des cultures. In M. Henzelin \& R. Roth, Le droit pénal à l'épreuve de l'internationalisation (69-118), Paris-Genève-Bruxelles: LGDJ-Georg-Bruylant.

Hughes, R. A. (2000). Health, place and British prisons. Healt $\mathfrak{G}$ Place, 6 (1), 57-62.

Kaminski, D. (2002). Les droits des détenus au Canada et en Angleterre: entre révolution normative et légitimation de la prison. In O. De Schutter \& D. Kaminski (eds.), L'institution du droit pénitentiaire. Enjeux de la reconnaissance des droits des détenus (91-112). Paris-Bruxelles: LGDJ-Bruylant.

Kaminski, D. (2006). Un nouveau sujet de droit pénal? In Th. Moreau \& F. Digneffe (eds.), La responsabilité et la responsabilisation dans la justice pénale (323-342). Bruxelles: De Boeck-Larcier.

Keslassy, E. (2000). Le libéralisme de Tocqueville à l'épreuve du paupérisme. Paris: L'Harmattan.

King, R. D., \& Morgan, R. (1980). The Future of the Prison System. Farnborough: Gower.

Landenne, Ph. (2008). Peines en prison: l’addition cachée. Bruxelles: Larcier.

Landreville, P. (1976). Les détenus et les droits de 1'homme. Criminologie, 9 (1-2), 107-117.

Landreville, P. (1978). Les détenus et les droits de l'homme. Revue de droit pénal et de criminologie, 387-400.

Law and Justice Foundation (2008). Taking justice into custody: research into prisoners' and ex-prisoners' legal needs. New South Wales (Australia): http:// www.lawfoundation.net.au/ljf/app/E3C05A0DFA5A6E08CA25748D000 0E890.html.

Lemire, G. (1990). Anatomie de la prison. Montréal: Presses de 1'Université de Montréal.

Lemire, G. (1991). Vingt ans de droits des détenus au Québec. Criminologie, 24 (1), 63-76.

Macquet, C. (2003). L'échange social et la régulation des déviances à l'aube de la (possible) postmodernité. In D. Kaminski (ed.), L'usage pénal des drogues (299-323). Bruxelles: De Boeck.

Maes, E. (2009). Van gevangenisstraf naar vrijheidsstraf. 200 jaar gevangeniswezen. Antwerpen-Apeldoorn: Maklu. 
Mary, Ph. (2006). À propos des similitudes entre «guerre anti-terroriste» et «lutte contre la délinquance urbaine». Revue de Science criminelle et de droit pénal comparé, 2, 476-484.

Mary, Ph., Bartholeyns, F., \& Béghin, J. (2006). La prison en Belgique: de l'institution totale aux droits des détenus? Déviance et Société, 30 (3), 389404.

Morgan, G. (1999). Images de l'organisation. Québec: Les Presses de l'Université Laval.

Ost, F. (2007). Quand l'enfer se pave de bonnes intentions. In Y. Cartuyvels, H. Dumont, F. Ost, M. van de Kerchove \& S. Van Drooghenbroeck (eds.), Les droits de l'bomme, bouclier ou épée du droit pénal? (7-21). Bruxelles: Publications des FUSL.

Philippe, F. (2008). Espace squat: la métamorphose de soi dans la culture de la rue. Mémoire de licence en criminologie, Louvain-la-Neuve: Université catholique de Louvain, École de criminologie.

Poncela, P. (2007). La logique modale de la peine dans la jurisprudence de la Cour européenne des droits de l'homme. In Y. Cartuyvels, H. Dumont, F. Ost, M. van de Kerchove \& S. Van Drooghenbroeck (eds.), Les droits de l'bomme, bouclier ou épée du droit pénal? (363-376). Bruxelles: Publications des FUSL.

Poncela, P., \& Lascoumes, P. (1998). Réformer le code pénal. Où est passé l'architecte? Paris: PUF.

Rancière, J. (1995). La Mésentente, Philosophie et Politique. Paris: Galilée, La philosophie en effet.

Reynaert, P. (2004). La prison entre immobilisme et mouvement perpétuel. In D. Kaminski \& M. Kokoreff (eds.), Sociologie pénale: système et expérience. Pour Claude Faugeron (235-255). Ramonville Saint-Agne: Eres.

Robert, L. (2007). Een deurwaarder in de gevangenis. Naar een 'cachot pour dettes'? Fatik. Tijdschrift voor Strafbeleid en Gevangeniswezen, 116, 16-20.

Rostaing, C. (2007). Processus de judiciarisation carcérale: le droit en prison, une ressource pour les acteurs. Droit et Société, 67, 577-595.

Rusche, G., Kirchheimer, O. (1939/1994). Peine et structure sociale. Texte présenté et établi par R. Lévy et H. Zander. Paris: Éditions du Cerf.

Scheingold, S. A. (1974). The Politics of Rights. Lawyers, Public Policy and Political Change. New Haven-London: Yale University Press.

Sennett, R. (2008). La culture du nouveau capitalisme. Paris: Hachette Littératures.

Shea, E. (2005). Les paradoxes de la normalisation du travail pénitentiaire en France et en Allemagne. Déviance et Société, 29 (3), 349-365.

Snacken, S. (2001). Belgium. In D. Van Zyl Smit \& F. Dünkel (eds.), Imprisonment Today and Tomorrow: International Perspectives on Prisoners' Rights and Prison Conditions (32-81). The Hague: Kluwer Law International.

Snacken, S. (2002). 'Normalisation' dans les prisons: concept et défis. L'exemple de l'Avant-projet de loi pénitentiaire belge. In O. De Schutter \& D. Kaminski (eds.), L'institution du droit pénitentiaire. Enjeux de la reconnaissance des droits des détenus (133-152). Paris-Bruxelles: LGDJ-Bruylant. 
Supiot, A. (2000). La contractualisation de la société. In Y. Michaud (ed.), Qu'est-ce que l'bumain? Vol. 2 (257-267). Paris: Odile Jacob.

Supiot, A. (2005). Homo juridicus. Essai sur les fondements anthropologiques du droit. Paris: Seuil.

Trombert, Chr. (2008). Less eligibility et activation: une mise en perspective de l'activation des politiques sociales par l'étude des principes antérieurs d'articulation du travail et de la protection. In Coordination des intermittents et précaires, Le gouvernement des individus, Université ouverte (20072008), http://www.cip-idf.org/article.php3 ?id_article=3738.

Tulkens, F. (2002). Droits de l'homme et prison. In O. De Schutter \& D. Kaminski (eds.), L'institution du droit pénitentiaire, enjeux de la reconnaissance de droits aux détenus (249-285). Paris-Bruxelles: LGDJ-Bruylant.

Tulkens, F., van de Kerchove, M. (2005). Les droits de l'homme: bonne ou mauvaise conscience du droit pénal? In F. Verbruggen, R. Verstraeten, R. Van Daele \& B. Spriet (eds.), Strafrecht als roeping. Liber amicorum Lieven Dupont. Vol. 2 (949-968). Leuven: Leuven University Press.

van de Kerchove, M. (2007). Les caractères et les fonctions de la peine. In Y. Cartuyvels, H. Dumont, F. Ost, M. van de Kerchove \& S. Van Drooghenbroeck (eds.), Les droits de l'homme, bouclier ou épée du droit pénal? (337-361). Bruxelles: Publications des FUSL.

Van Drooghenbroeck, S. (2007). Droit pénal et droits de l'homme: le point de vue de la Cour européenne des droits de l'homme. In Y. Cartuyvels, H. Dumont, F. Ost, M. van de Kerchove \& S. Van Drooghenbroeck (eds.), Les droits de l'homme, bouclier ou épée du droit pénal? (75-110). Bruxelles: Publications des FUSL.

ABSTRACT - Answering to the invitation of the journal Criminologie for its fortieth birthday, this conference, given on November $7^{\text {th }} 2008$, questions the contemporary promotion of the prisoners' rights in a socio-political context which gives a good place to less eligibility. A really normalizing effectivity of the prisoners' rights asks for the lifting of political and cultural obstacles which today impoverish their impact.

KEYWORDS • Prisoners' rights, normalization, less eligibility.

RESUMEN - Elaborada a petición de la revista Criminologie en ocasión de su cuadragésimo aniversario, esta conferencia dictada el 7 de noviembre de 2008 cuestiona la promoción contemporánea de los derechos de los presos en un contexto sociopolítico que privilegia la menor elegibilidad. Una real estandarización de los derechos de los prisioneros requeriría de la eliminación de los obstáculos políticos y culturales que en la actualidad empobrecen su efecto.

PALABRAS CLAVE • Derechos de los presos, estandardización, menor elegibilidad. 